\title{
Public Preparedness - Rethinking the Unthinkable
}

\section{James J James, MD, DrPH}

I n August 22 and 23 the National Academies put on a workshop titled "Exploring Medical and Public Health Preparedness for a Nuclear Incident" at which I served as a panelist.

The National Academy of Sciences (NAS) plans a future publication on the presentations, conclusions and recommendations and I will not attempt to provide specific details from the workshop herein. I will provide an overview of what I interpreted the objectives of the meeting to be.

Underscoring the overwhelming number of expected casualties from a nuclear event ${ }^{1}$ and contrasting those numbers against capabilities and capacities the shortfalls are identified and presented and future efforts to overcome them are identified.

The workshop, in essence, was eerily reminiscent of the work presented in a special issue of DMPHP Nuclear Preparedness (March, 2011) ${ }^{2}$ for which Richard Hatchett served as guest editor.

In preparing for my presentation I reviewed both the special issue and the topics being addressed at the NAS workshop. It struck me that we were doing excellent work and making real progress in increasing capabilities and capacities, but felt that we were not looking at maximizing these variables indirectly through decreasing the number of casualties, both in terms of number and severity. This is not a novel concept $\mathrm{t}^{3}$ but optimizing it has not been well thought out, and has certainly not been addressed through a systematic approach. Providing and championing such an approach would, to me, be an ideal initial "position" for the Society to take.

One model that we can incorporate in our efforts would be the adoption of the classical preventative template of $1^{\circ}, 2^{0}$ and $3^{0}$ prevention. I realize there are proponents of two other levels of preventionprimal and quaternary but initially it might be best to focus on the basic model that has stood the test of time; at a later date such additional levels can be addressed, if applicable. One other assumption that I feel we should make in our initial considerations is that the prevention of a nuclear event may not even be possible at all, let alone through initiatives on the part of the health system. Therefore, for the proposed model, the healthcare preventative levels are defined post event and our particular focus will be on a primary prevention. To facilitate further understanding and collaboration we utilize a modified Haddon matrix.

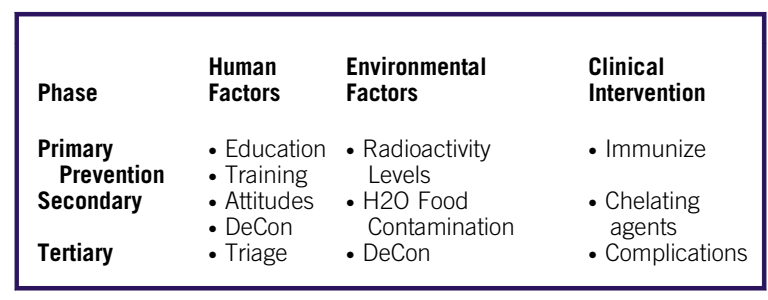

It should be noted that the entries in the grid are preliminary and should not be interpreted as definitive. What we should strive for is developing a tool that will incorporate knowledge from the broad spectrum of professionals of all disciplines involved in this arena. In its final, or mature, form, it should serve as a template so that we can better differentiate often competing viewpoints in order to achieve greater efficiency in our research and development efforts by helping better identify priorities and channel efforts. That cell of the matrix that seems both most natural as a focus for SDMPH and most in need of critical analytical input is the Human Factors area of primary prevention. I sincerely believe that a prepared citizenry will be the single best mechanism to decrease casualties from a nuclear event and thus maximize our existing capacities and capabilities. Further development of this concept will be the centerpiece of our Annual Meeting and help formulate our first position.

\section{REFERENCES}

1. Bell WC, Dallas CE. Vulnerability of populations and the urban health care systems to nuclear weapon attack-examples from four American cities. Int J Health Geogr. 2007;6:5.

2. Disaster Med Public Health Preparedness. 2011;5(S1):S7-S158. Special issue.

3. Coleman C, Knebel A, Hick J, et al. Scarce Resources for Nuclear Detonation: Project Overview and Challenges. Disaster Med Public Health Preparedness. 2011;5(S1):S13-S19. doi: 10.1001/dmp.2011.15 